Relativistic scalar Aharonov-Bohm scattering

This article has been downloaded from IOPscience. Please scroll down to see the full text article.

2000 J. Phys. A: Math. Gen. 335521

(http://iopscience.iop.org/0305-4470/33/31/307)

View the table of contents for this issue, or go to the journal homepage for more

Download details:

IP Address: 200.130.19.138

The article was downloaded on 24/07/2013 at 19:20

Please note that terms and conditions apply. 


\title{
Relativistic scalar Aharonov-Bohm scattering
}

\author{
M Gomes $\dagger$, J M C Malbouisson $\ddagger$ A G Rodrigues $\dagger$ and A J da Silva $\dagger$ \\ $\dagger$ Instituto de Física, Universidade de São Paulo, Caixa Postal 66318, 05315-970 São Paulo, SP \\ Brazil \\ \$ Instituto de Física, Universidade Federal da Bahia, Campus de Ondina, 40210-340 Salvador, \\ BA, Brazil
}

Received 28 April 2000

\begin{abstract}
We discuss the scattering of relativistic spin zero particles by an infinitely long and arbitrarily thin solenoid. The exact solution of the first-quantized problem can be obtained as a mimic of the nonrelativistic case, either in the original Aharonov-Bohm way or by using the Berry magnetization scheme. The perturbative treatment is developed in the Feshbach-Villars two-component formalism for the Klein-Gordon equation and it is shown that it also requires renormalization as in the Schrödinger counterpart. The results are compared with those of the field theoretical approach, which corresponds to the two-body sector of the scalar Chern-Simons theory.
\end{abstract}

\section{Introduction}

The Aharonov-Bohm (AB) effect [1], the scattering of a charged particle by an infinitely long and arbitrarily thin solenoid, presents a very peculiar situation of nonrelativistic (NR) quantum dynamics, with charged particles feeling the vector potential in regions where the electromagnetic field is null. It is an exactly solvable quantum mechanical problem [1,2], which, due to the singular nature of the potential, requires the use of renormalization procedures to make its perturbative treatment meaningful. In fact, as noticed by Corinaldesi and Rafeli [3], the bare perturbation theory leads to an incomplete result in the Born approximation and to a divergent one in second order. For spinless particles in quantum mechanics, the necessary renormalization is accomplished by adding a delta function potential [4], while in the second quantized version it is implemented by introducing a $\phi^{4}$ self-interaction, with an appropriate strength, in a scalar Chern-Simons Lagrangian [5-7].

In this paper, we discuss the relativistic scalar $\mathrm{AB}$ scattering, that is the scattering of a relativistic charged spin zero particle by a thin fixed solenoid from the viewpoint of the first quantization, comparing with the analysis in the framework of the field theory [8-10]. In section 2 , it is shown that the problem can be solved exactly through a mimic of either the original $\mathrm{AB}$ solution or the Berry magnetization scheme. We then develop (section 3) a perturbative analysis of the Klein-Gordon equation in the presence of the solenoid using the Feshbach-Villars two-component formalism and show that the bare perturbation treatment presents problems similar to those of the NR case and possesses an analogous renormalization. In section 4 , the results of the field theoretical perturbative approach, corresponding to the two-body sector of the Chern-Simons theory, are presented and compared with the scattering amplitude obtained in the relativistic quantum mechanics. Finally, some conclusions are outlined. 


\section{Exact solution in the framework of the first quantization}

The Klein-Gordon equation in the presence of an external electromagnetic field, fixing the Coulomb gauge $(\boldsymbol{\nabla} \cdot \boldsymbol{A}=0)$, can be written (in natural units, $\hbar=c=1$ ) as

$$
\left(\partial_{t}^{2}-\nabla^{2}+m^{2}+\mathcal{U}\right) \phi=0
$$

where

$$
\mathcal{U}=e A^{0}-\mathrm{i} 2 e \boldsymbol{A} \cdot \nabla+e^{2} \boldsymbol{A}^{2} .
$$

For an ideal AB solenoid (a line carrying magnetic flux $\Phi$ ) at the origin, the magnetic field

$$
\boldsymbol{B}=\Phi \delta(\boldsymbol{r}) \hat{z}
$$

with $r=\left(x^{1}, x^{2}, 0\right)$, and one may choose

$$
A^{0}=A^{3}=0 \quad A^{i}=-\frac{\Phi}{2 \pi} \frac{\epsilon^{i j} x^{j}}{r^{2}} \quad i=1,2
$$

where $\epsilon^{i j}$ is the anti-symmetric symbol $\left(\epsilon^{12}=1\right)$. The potential (2) then becomes

$$
\mathcal{U}_{\text {Sol }}=-\mathrm{i}\left(\frac{e \Phi}{\pi}\right) \frac{r \times \nabla}{r^{2}}+\left(\frac{e \Phi}{\pi}\right)^{2} \frac{1}{r^{2}}
$$

showing that the problem, owing to the symmetry, is actually a two-dimensional one.

Considering a particle with (positive) energy $w_{p}$ ( $\boldsymbol{p}$ in the $x^{1}$ negative direction), the wavefunction can be separated as $\phi(\boldsymbol{r}, t)=\exp \left(-\mathrm{i} w_{p} t\right) \phi(\boldsymbol{r})$ and (1) reduces (in cylindrical coordinates) to

$$
\left[\frac{1}{r} \frac{\partial}{\partial r}\left(r \frac{\partial}{\partial r}\right)+\frac{1}{r^{2}}\left(\frac{\partial}{\partial \theta}+\mathrm{i} \alpha\right)^{2}+p^{2}\right] \phi(r, \theta)=0
$$

where $\alpha=e \Phi / 2 \pi$ is the magnetic flux parameter and $p^{2}=w_{p}^{2}-m^{2}$. This equation coincides with the one solved by Aharonov and Bohm for the NR particle if one replaces the dispersion relation by $p^{2}=2 m E, E$ being the NR energy. Thus, the exact solution of (6), vanishing when $r \rightarrow 0$ as required by the 'impenetrability' condition, is given by [1]

$$
\phi(r, \theta)=\sum_{l=-\infty}^{+\infty}(-\mathrm{i})^{|l-\alpha|} J_{|l-\alpha|}(p r) \exp (\mathrm{i} l \theta)
$$

where $J_{|l-\alpha|}$ denotes a Bessel function of the first kind.

This exact solution of the Klein-Gordon equation in the presence of the solenoid can also be obtained, in a rather distinct way, by applying the Berry magnetization scheme [2] to its solution in the absence of the flux line, corresponding to the incident plane wave. Using the Fourier expansion of a plane wave,

$$
\phi_{0}(r, \theta)=\exp [-\mathrm{i} p r \cos (\theta)]=\sum_{l=-\infty}^{+\infty}(-\mathrm{i})^{|l|} J_{|l|}(p r) \exp (\mathrm{i} l \theta)
$$

and the Poisson summation formula

$$
\sum_{l=-\infty}^{+\infty} f(l)=\sum_{m=-\infty}^{+\infty} \int_{-\infty}^{+\infty} \mathrm{d} \eta f(\eta) \exp (2 \pi \mathrm{i} m \eta)
$$

one obtains the whirling-wave expansion of the free solution

$$
\begin{aligned}
& \phi_{0}(r, \theta)=\sum_{m=-\infty}^{+\infty} w_{m}(r, \theta) \\
& w_{m}(r, \theta)=\int_{-\infty}^{+\infty} \mathrm{d} \eta \exp \left(-\frac{1}{2} \mathrm{i} \pi|\eta|\right) J_{|\eta|}(p r) \exp [\mathrm{i} \eta(\theta+2 \pi m)] .
\end{aligned}
$$


Notice that $w_{m}$ is not single valued but satisfies $w_{m}(r, \theta+2 \pi)=w_{m+1}(\theta)$, therefore guaranteeing that $\phi_{0}(r, \theta)$ has a unique value.

The ingenious idea of Berry [2] to rescue the Dirac magnetization prescription, by which incorporating the phase factor $\exp \left(\mathrm{i} q \int_{r_{0}}^{r} A\left(\boldsymbol{r}^{\prime}\right) \cdot \mathrm{d} \boldsymbol{r}^{\prime}\right)$ into the free wavefunction would lead to the solution in the presence of the magnetic field, and to produce a single-valued wavefunction, was to apply the Dirac procedure to each whirling wave separately and to resum the 'magnetized' expansion. Doing so, one finds

$$
\begin{aligned}
& \phi_{0}^{D}(r, \theta)=\sum_{m=-\infty}^{+\infty} w_{m}^{D}(r, \theta) \\
& w_{m}^{D}(r, \theta)=\int_{-\infty}^{+\infty} \mathrm{d} \eta \exp \left(-\frac{1}{2} \mathrm{i} \pi|\eta|\right) J_{|\eta|}(p r) \exp [\mathrm{i}(\eta+\alpha)(\theta+2 \pi m)]
\end{aligned}
$$

and, making the change of variables $\eta^{\prime}=\eta+\alpha$, the inverse Poisson transform leads to the exact solution (7). Notice that the Berry procedure relies on the fact that the free solution is a plane wave and so it can be applied equally well to both relativistic and NR AB scattering. An extension of the whirling-wave formulation of Berry to the case of the Dirac equation has been presented recently [11].

The $\mathrm{AB}$ scattering amplitude can be obtained by analysing the asymptotic behaviour of the exact solution (7). It can be shown that $[1,3]$

$$
\phi(r, \theta) \stackrel{r \gg 1}{\longrightarrow} \mathrm{e}^{-\mathrm{i} p r \cos \theta}+\mathrm{e}^{\mathrm{i} \pi / 4} \mathcal{A}_{\mathrm{AB}}(|p|, \theta) \frac{\mathrm{e}^{\mathrm{i} p r}}{\sqrt{r}}
$$

where the scattering amplitude is given by

$$
\mathcal{A}_{\mathrm{AB}}(|\boldsymbol{p}|, \theta)=-\frac{\mathrm{i}}{\sqrt{2 \pi p}} \sin (\pi \alpha)\left[\tan \left(\frac{\theta}{2}\right)-\mathrm{i} \operatorname{sgn}(\alpha)\right]
$$

with $\operatorname{sgn}(\alpha)=|\alpha| / \alpha$. It should be remarked that the exact solution, and consequently the $\mathrm{AB}$ scattering amplitude, can be also obtained within the two-component formalism, which is employed to construct the perturbative expansion of the $S$ matrix in the next section.

\section{Perturbative analysis of the relativistic AB scattering}

In order to be able to use the standard perturbation theory one has to cast the Klein-Gordon equation as a differential equation of first order in time, that is in a form similar to the Schrödinger equation. This can be done following the prescription below.

\subsection{Two-component formalism of the Klein-Gordon equation}

In the Feshbach-Villars representation [12], the Klein-Gordon wavefunction in the presence of an external field $A^{\mu}$ is written in the form

$$
\Psi=\left(\begin{array}{l}
\chi \\
\zeta
\end{array}\right) \longleftrightarrow\left\{\begin{array}{l}
\chi=\frac{1}{\sqrt{2 m}}\left[\mathrm{i} \partial_{t} \phi-\left(e A^{0}-m\right) \phi\right] \\
\zeta=\frac{1}{\sqrt{2 m}}\left[-\mathrm{i} \partial_{t} \phi+\left(e A^{0}+m\right) \phi\right] .
\end{array}\right.
$$

This two-component wavefunction satisfies a Schrödinger-like equation,

$$
\mathrm{i} \partial_{t} \Psi=H \Psi
$$

with the 'Hamiltonian' given by

$$
H=\left(m-\frac{(\boldsymbol{\nabla}-\mathrm{i} e \boldsymbol{A})^{2}}{2 m}\right) \tau_{3}-\frac{(\boldsymbol{\nabla}-\mathrm{i} e \boldsymbol{A})^{2}}{2 m} \mathrm{i} \tau_{2}+e A^{0}
$$


where $\tau_{i}$ are the Pauli matrices. Although $H$ is not Hermitian, $\tau_{3} H^{\dagger} \tau_{3}=H$ and the norm $\int \mathrm{d} x \Psi^{\dagger} \tau_{3} \Psi$ is conserved, i.e. $\tau_{3}$ plays the role of a metric tensor.

This formalism allows the use of perturbation theory in the standard way and we make the partition $H=H_{0}+H_{\text {int }}=H_{0}+V+H_{1}\left[\tau_{3}+i \tau_{2}\right]$ where $V=e A^{0}$ and

$$
\begin{aligned}
& H_{0}=\left(m-\frac{\nabla^{2}}{2 m}\right) \tau_{3}-\frac{\nabla^{2}}{2 m} \mathrm{i} \tau_{2} \\
& H_{1}=\left(\frac{\mathrm{i} e}{2 m}[\boldsymbol{\nabla} \cdot \boldsymbol{A}+\boldsymbol{A} \cdot \boldsymbol{\nabla}]+\frac{e^{2}}{2 m} \boldsymbol{A}^{2}\right) .
\end{aligned}
$$

The free positive- and negative-energy solutions are given by

$$
\begin{aligned}
& \Psi_{p}^{(+)}(\boldsymbol{x}, t)=\mathrm{e}^{-\mathrm{i} w_{p} t} \Psi_{p}^{(+)}(\boldsymbol{x})=\frac{1}{2 \pi} \frac{w_{p}+m}{\sqrt{4 m w_{p}}}\left(\begin{array}{c}
1 \\
\frac{m-w_{p}}{m+w_{p}}
\end{array}\right) \mathrm{e}^{\mathrm{i} p \cdot \boldsymbol{x}-\mathrm{i} w_{p} t} \\
& \Psi_{p}^{(-)}(\boldsymbol{x}, t)=\mathrm{e}^{+\mathrm{i} w_{p} t} \Psi_{p}^{(-)}(\boldsymbol{x})=\frac{1}{2 \pi} \frac{w_{p}+m}{\sqrt{4 m w_{p}}}\left(\begin{array}{c}
\frac{m-w_{p}}{m+w_{p}} \\
1
\end{array}\right) \mathrm{e}^{-\mathrm{i} p \cdot x+\mathrm{i} w_{p} t}
\end{aligned}
$$

which satisfy the normalization conditions

$$
\int \mathrm{d} x \Psi_{p^{\prime}}^{( \pm)} \tau_{3} \Psi_{p}^{( \pm)}= \pm \delta\left(p^{\prime}-p\right) .
$$

Notice that the Berry magnetization procedure can be applied to the positive-energy solution (21), leading to the exact solution described in the last section and thus to the $\mathrm{AB}$ scattering amplitude (15).

In fact, the two-component formalism is the appropriate scenario to implement the perturbative treatment of the scalar $\mathrm{AB}$ scattering in the framework of the relativistic quantum mechanics. The $S$ matrix elements can be calculated through the formula

$$
S_{f i}=\left\langle f\left|\mathrm{~T} \exp \left[-\mathrm{i} \int \mathrm{d} t H_{I}(t)\right]\right| i\right\rangle
$$

where $\mathrm{T}$ represents the time ordering operator and $H_{I}(t)$ is the interaction picture of the interaction Hamiltonian $H_{\mathrm{int}}$, from which we can define the scattering amplitude $\mathcal{A}_{f i}$ as

$$
S_{f i}=-2 \pi \mathrm{i} \delta\left(w_{f}-w_{i}\right) \mathcal{A}_{f i} .
$$

\subsection{The bare scattering amplitude}

The $S$ matrix, expressing the scattering of a positive-energy particle, in the first Born approximation, becomes

$$
S_{f i}^{(1)}=-\mathrm{i} \int \mathrm{d} t \mathrm{~d} \boldsymbol{x} \Psi_{p^{\prime}}^{\dagger}(\boldsymbol{x}) \tau_{3} H_{\mathrm{int}}(\boldsymbol{x}, \boldsymbol{t}) \Psi_{p}(\boldsymbol{x})=-\mathrm{i} 2 \pi \delta\left(w_{p^{\prime}}-w_{p}\right) \mathcal{A}_{f i}^{(1)}
$$

with the reduced amplitude given by

$$
\mathcal{A}_{f i}^{(1)}=\int \mathrm{d} \boldsymbol{x} \mathrm{e}^{-\mathrm{i} p^{\prime} \cdot \boldsymbol{x}}\left[\frac{m}{w_{p}} H_{1}(\boldsymbol{x}, \boldsymbol{\nabla})+V(\boldsymbol{x})\right] \mathrm{e}^{\mathrm{i} p \cdot \boldsymbol{x}} .
$$

Considering the Coulomb gauge, $\boldsymbol{\nabla} \cdot \boldsymbol{A}=0$, and for the moment neglecting the higher-order term $e^{2} \boldsymbol{A}^{2}$, one obtains

$\mathcal{A}_{f i}=\int \mathrm{d} \boldsymbol{x}\left[-\frac{e}{w_{p}} \boldsymbol{A}(\boldsymbol{x}) \cdot \boldsymbol{p}+V(\boldsymbol{x})\right] \mathrm{e}^{-\mathrm{i}\left(p^{\prime}-p\right) \cdot \boldsymbol{x}}=-\frac{e}{w_{p}} \tilde{\boldsymbol{A}}(\boldsymbol{q}) \cdot \boldsymbol{p}+\tilde{V}(\boldsymbol{q})$

where $q=p^{\prime}-p$. 
For the $\mathrm{AB}$ potential, $V=0$ and

$\tilde{A}^{i}(\boldsymbol{q})=\frac{\mathrm{i} \Phi}{2 \pi} \epsilon^{i j} \lim _{\lambda \rightarrow 0} \partial_{q^{j}} \int \mathrm{d} \boldsymbol{r} \frac{\mathrm{e}^{\mathrm{i} \boldsymbol{q} \cdot \boldsymbol{r}}}{\boldsymbol{r}^{2}+\lambda^{2}}=\mathrm{i} \Phi \epsilon^{i j} \lim _{\lambda \rightarrow 0} \partial_{q^{j}} K_{0}(\lambda|\boldsymbol{q}|)=-\mathrm{i} \Phi \frac{\epsilon^{i j} q^{j}}{\boldsymbol{q}^{2}}$

$K_{0}$ denoting the modified Bessel function, and therefore the reduced amplitude is given by

$$
\mathcal{A}_{f i}^{(1)}=-\frac{\mathrm{i} e \Phi}{w_{p}} \frac{\boldsymbol{p} \times \boldsymbol{q}}{\boldsymbol{q}^{2}}=-\frac{\mathrm{i} e \Phi}{2 w_{p}} \cot \left(\frac{\theta_{S}}{2}\right)
$$

where $\theta_{S}$ is the scattering angle. Notice that, since $\theta_{S}$ is the angle between the incoming $(\boldsymbol{p})$ and the outgoing $\left(\boldsymbol{p}^{\prime}\right)$ momenta, it is related to the $\mathrm{AB}$ polar angle by $\theta_{S}=\pi-\theta$. One immediately notices the absence of the nonanalytic term occurring in the first order of the expansion of the exact result (15).

To calculate higher-order contributions one should be aware of the fact that, since the interaction with the external field contains terms of order $e$ and $e^{2}$, the perturbative expansion in the coupling constant does not correspond 'order by order' to the Born expansion. Thus, to find the second-order contribution to the amplitude, besides the contribution from the second term of the expansion (24), one has to account for the $A^{2}$ term present in the first Born approximation; one obtains

$$
\mathcal{A}_{f i\left(\boldsymbol{A}^{2}\right)}^{(2)}=\int \mathrm{d} \boldsymbol{r} \mathrm{e}^{-\mathrm{i} \boldsymbol{p}^{\prime} \cdot \boldsymbol{r}}\left[\frac{m}{w_{p}} \frac{e^{2}}{2 m} \boldsymbol{A}^{2}(\boldsymbol{r})\right] \mathrm{e}^{\mathrm{i} p \cdot r}=\frac{m}{w_{\boldsymbol{p}}} \frac{e^{2}}{2 m} \tilde{\mathcal{C}}(\boldsymbol{q})
$$

where the Fourier transform of $\boldsymbol{A}^{2}$,

$\tilde{\mathcal{C}}(\boldsymbol{q})=\left(\frac{\Phi}{2 \pi}\right)^{2} \int \mathrm{d} \boldsymbol{r} \frac{\mathrm{e}^{-\mathrm{i} \boldsymbol{q} \cdot \boldsymbol{r}}}{r^{2}}=\left(\frac{\Phi}{2 \pi}\right)^{2} \lim _{\lambda \rightarrow 0} \int \mathrm{d} \boldsymbol{r} \frac{\mathrm{e}^{-\mathrm{i} \boldsymbol{q} \cdot \boldsymbol{r}}}{r^{2}+\lambda^{2}}=\left(\frac{\Phi}{2 \pi}\right)^{2} \lim _{\lambda \rightarrow 0} K_{0}(\lambda|\boldsymbol{q}|)$

leads to a divergent contribution. Notice that it is precisely due to the absence of the $\boldsymbol{A}^{2}$ term in the interaction of the spin-half particles with the field that no divergence occurs in that case; furthermore the Pauli magnetic interaction naturally makes the first Born approximation correct [13]. Wishing to be able to suppress the divergence of the scalar case, we keep the regularized contribution

$\mathcal{A}_{f i\left(A^{2}\right)}^{(2) \operatorname{Reg}}=\frac{e^{2} \Phi^{2}}{4(2 \pi)^{3}} \frac{1}{w_{p}}\left\{-\ln \left(\lambda^{2}\right)-\ln \left(p^{2}\right)-\ln \left[2\left(1-\cos \theta_{S}\right)\right]+2(\ln 2-\gamma)\right\}$

where $\gamma$ is the Euler constant.

The other second-order contribution originates from the second Born approximation,

$S_{f i}^{(2)}=-\iint \mathrm{d} t_{1} \mathrm{~d} t_{2} \theta\left(t_{2}-t_{1}\right) \int \mathrm{d} \boldsymbol{r}_{2} \Psi_{p^{\prime}}^{(+) \dagger}\left(\boldsymbol{r}_{\mathbf{2}}\right) \tau_{3} H_{\mathrm{int}}\left(\boldsymbol{r}_{\mathbf{2}}, t_{2}\right) H_{\mathrm{int}}\left(\boldsymbol{r}_{\mathbf{2}}, t_{1}\right) \Psi_{\boldsymbol{p}}^{(+)}\left(\boldsymbol{r}_{\mathbf{2}}\right)$

considering only the part of the interaction Hamiltonian which is first order in $e$. Using the completeness relation

$$
\int \mathrm{d} k\left[\Psi_{k}^{(+)}\left(r_{1}\right) \Psi_{k}^{(+) \dagger}\left(r_{2}\right) \tau_{3}-\Psi_{k}^{(-)}\left(r_{1}\right) \Psi_{k}^{(-) \dagger}\left(r_{2}\right) \tau_{3}\right]=I \delta\left(r_{1}-r_{2}\right)
$$

and the identity

$$
\theta(t) E^{n} \mathrm{e}^{-\mathrm{i} E t}=\frac{1}{2 \pi \mathrm{i}} \int \mathrm{d} \omega \frac{\omega^{n} \mathrm{e}^{-\mathrm{i} \omega t}}{E-\omega-\mathrm{i} \epsilon}
$$

one obtains

$$
S_{f i(\boldsymbol{A} \cdot \boldsymbol{p})}^{(2)}=-2 \pi \mathrm{i} \delta\left(w_{f}-w_{i}\right) \mathcal{A}_{f i(\boldsymbol{A} \cdot \boldsymbol{p})}^{(2)}
$$


with

$$
\begin{aligned}
\mathcal{A}_{f i(\boldsymbol{A} \cdot \boldsymbol{p})}^{(2)}=- & \frac{e^{2}}{(2 \pi)^{4}} \frac{1}{w_{p}} \int \frac{\mathrm{d} \boldsymbol{k}}{\omega_{k}}\left\{\frac{\left[\boldsymbol{k} \cdot \tilde{\boldsymbol{A}}\left(\boldsymbol{p}^{\prime}-\boldsymbol{k}\right)\right][\boldsymbol{p} \cdot \tilde{\boldsymbol{A}}(\boldsymbol{k}-\boldsymbol{p})]}{\omega_{p}-\omega_{k}+\mathrm{i} \epsilon}\right. \\
& \left.-\frac{\left[\boldsymbol{k} \cdot \tilde{\boldsymbol{A}}\left(\boldsymbol{p}^{\prime}+\boldsymbol{k}\right)\right][\boldsymbol{p} \cdot \tilde{\boldsymbol{A}}(\boldsymbol{k}+\boldsymbol{p})]}{\omega_{p}+\omega_{k}+\mathrm{i} \epsilon}\right\}
\end{aligned}
$$

where $w_{k}=\sqrt{\boldsymbol{k}^{2}+m^{2}}$. From (29), it follows that $\boldsymbol{k} \cdot \tilde{\boldsymbol{A}}(\boldsymbol{p})=\mathrm{i} \Phi(\boldsymbol{k} \times \boldsymbol{p}) / p^{2}$, so the contribution (38) to the second-order amplitude is given by

$$
\begin{aligned}
\mathcal{A}_{f i(\boldsymbol{A} \cdot \boldsymbol{p})}^{(2)} & =\frac{e^{2} \Phi^{2}}{(2 \pi)^{4}} \frac{1}{w_{p}} \int \mathrm{d} \boldsymbol{k} \frac{\left(\boldsymbol{k} \times \boldsymbol{p}^{\prime}\right)(\boldsymbol{k} \times \boldsymbol{p})}{\left(\boldsymbol{k}-\boldsymbol{p}^{\prime}\right)^{2}(\boldsymbol{k}-\boldsymbol{p})^{2}} \frac{2}{p^{2}-k^{2}+\mathrm{i} \epsilon} \\
& =\frac{e^{2} \Phi^{2}}{4(2 \pi)^{3}} \frac{1}{w_{p}}\left\{\ln \left[2\left(1-\cos \theta_{S}\right)\right]+\mathrm{i} \pi\right\}
\end{aligned}
$$

where the integral was performed as presented in [10]. The $\theta_{S}$ dependent part of this finite contribution is cancelled out with one of the terms of $\mathcal{A}_{f i\left(A^{2}\right)}^{(2)}$. One thus obtains, adding up both contributions, a divergent second-order term for the bare scattering amplitude. To recover a finite result, coinciding with the expansion of the exact amplitude, one has to implement some renormalization procedure.

\subsection{Perturbative renormalization}

To regain the correct perturbative expansion we have to search for an appropriate counterterm, an additional interaction, which should suppress the divergence of the second order and contributes in the lowest order, recovering the exact result. At first sight, one can try to follow the case of spin-half particles and look for a magnetic type of interaction to do the job. However, in the scalar case the magnetic interaction, given by $H_{\mathrm{mag}}=g \epsilon^{\mu \nu \rho} F_{v \rho} j_{\mu}$, where $F_{\nu \rho}$ is the field strength and $j_{\mu}$ the particle's current, can only furnish the correct result in the leading NR order [14], and thus cannot describe the full relativistic case. One of the reasons for this is the fact that such interaction does not have the same matrix structure in the twocomponent formalism as the $\boldsymbol{A}^{2}$ term, which is responsible for the divergence one wishes to eliminate.

The simplest additional interaction having the same matrix structure as the magnetic potential term that one can consider, leading to the same logarithmic divergence in second order, is a pure delta function external potential. In fact, by adding to (2) the term

$$
\mathcal{U}_{\text {(delta) }}=g \delta(\boldsymbol{r})
$$

one obtains, in first order, the contribution

$$
\mathcal{A}_{f i \text { (delta) }}^{(1)}=\frac{m g}{2} \frac{1}{w_{p}}
$$

while the cutoff regularized 'delta-delta' contribution to the second order becomes

$$
\begin{aligned}
\mathcal{A}_{f i \text { (delta) }}^{(2) \text { Reg }} & =\frac{m^{2} g^{2}}{(2 \pi)^{4}} \frac{1}{w_{p}} \int^{\Lambda^{2}} \mathrm{~d} \boldsymbol{k} \frac{1}{w_{p}-w_{k}+\mathrm{i} \epsilon} \\
& =\frac{m^{2} g^{2}}{4(2 \pi)^{3}} \frac{1}{w_{p}}\left\{-\ln \left(\Lambda^{2}\right)+\ln \left(p^{2}\right)-\mathrm{i} \pi\right\} .
\end{aligned}
$$

The crossing terms involving the delta and $\boldsymbol{A} \cdot \boldsymbol{p}$ interactions need not be considered; the sum of their contributions vanishes, a fact which can be inferred from symmetry arguments. 
Including these contributions from the delta potential, the first- and the regularized secondorder parts of the scattering amplitude become

$$
\begin{aligned}
\mathcal{A}_{f i}^{(1)}= & -\mathrm{i} \frac{e \Phi}{2 w_{p}} \cot \left(\frac{\theta_{S}}{2}\right)+\frac{m g}{2 w_{p}} \\
\mathcal{A}_{f i}^{(2)}= & \frac{1}{4(2 \pi)^{3}} \frac{1}{w_{p}}\left(m^{2} g^{2}-e^{2} \Phi^{2}\right)\left[\ln \left(p^{2}\right)-\mathrm{i} \pi\right] \\
& \quad \frac{1}{4(2 \pi)^{3}} \frac{1}{w_{p}}\left[-m^{2} g^{2} \ln \left(\Lambda^{2}\right)-e^{2} \Phi^{2}\left(\ln \left(\lambda^{2}\right)-2 \ln 2+2 \gamma\right)\right] .
\end{aligned}
$$

One sees then that the agreement with the expansion of the exact result can be reached if the strength of the delta interaction is fixed, satisfying

$$
m^{2} g^{2}=e^{2} \Phi^{2}=4 \pi^{2} \alpha^{2}
$$

and the cutoffs are adjusted so that

$$
\Lambda \lambda=2 \exp (-\gamma) \text {. }
$$

In doing so, the first-order term, multiplied by the appropriate kinematical factor, reproduces the correct result and the second-order term, proportional to $\alpha^{2}$, vanishes as it should.

\section{The field theoretical approach}

The scalar NR AB scattering corresponds, in the field theoretical approach, to the two-body sector of the theory of a Chern-Simons field coupled with a self-interacting scalar field for which the Lagrangian density is given by [7]

$$
\mathcal{L}_{\mathrm{NR}}=\psi^{*}\left(\mathrm{i} D_{t}+\frac{\boldsymbol{D}^{2}}{2 m}\right) \psi-\frac{v_{0}}{4}\left(\psi^{*} \psi\right)^{2}+\frac{\Theta}{2} \partial_{t} \boldsymbol{A} \times \boldsymbol{A}-\Theta A_{0} \nabla \times \boldsymbol{A}
$$

where $D_{t}=\partial_{t}+\mathrm{i} e A_{0}$ and $\boldsymbol{D}=\boldsymbol{\nabla}-\mathrm{i} e \boldsymbol{A}$ are covariant derivatives, $\Theta$ is the Chern-Simons parameter and $v_{0}$ is the bare self-coupling. The renormalized NR two-particle scattering amplitude, in the centre of mass (CM) frame, is given, up to one loop, by

$$
\mathcal{A}_{\mathrm{NR}}=-v-\mathrm{i} \frac{2 e^{2}}{m \Theta} \cot \theta+\frac{m}{8 \pi}\left(v^{2}-\frac{4 e^{4}}{m^{2} \Theta^{2}}\right)\left[\ln \left(\frac{\mu^{2}}{p^{2}}\right)+\mathrm{i} \pi\right]
$$

where $\mu$ is an arbitrary mass scale that breaks the scale invariance of the amplitude. By choosing the critical value $v_{c}^{+}=+2 e^{2} / m|\Theta|$, which corresponds to a repulsive quartic interaction, this amplitude reduces to the first-order $\left(e^{2}\right) \mathrm{AB}$ amplitude for identical particles, which is given by [7]

$$
\mathcal{F}_{\mathrm{AB}}(|p|, \theta)=-\mathrm{i} \frac{4 \pi \beta}{m}\left[\cot \theta_{S}-\mathrm{i} \operatorname{sgn}(\beta)\right]
$$

where $\beta=e^{2} / 2 \pi \Theta$ coincides with the flux parameter $\alpha$ if the identification $\Theta=e / \Phi$ is made; this symmetrized amplitude is the same, adjusting the kinematical factor, as the one obtained from the first-order expansion of the exact NR result (15).

It has been shown that the relativistic Chern-Simons theory,

$$
\mathcal{L}=\left(D_{\mu} \phi\right)^{*}\left(D^{\mu} \phi\right)-m^{2} \phi^{*} \phi-\frac{\lambda}{4}\left(\phi^{*} \phi\right)^{2}+\frac{\Theta}{2} \epsilon_{\sigma \mu \nu} A^{\sigma} \partial^{\mu} A^{\nu}
$$

reduces to the NR case in the leading approximation [8]. We have calculated in this theory the $|\boldsymbol{p}| / m$ expansion of the two-particle amplitude, up to one-loop order, using an intermediate cutoff procedure introduced in [15]. The renormalized CM amplitude, including the factor 
$1 / 4 w_{p}^{2}$ (where $w_{p}=\sqrt{m^{2}+p^{2}}$ ) which makes the states have the same normalization as in the NR case, can be written as $\mathcal{A}=\mathcal{A}^{(0)}+\mathcal{A}^{(1)}$ [9], where

$$
\mathcal{A}^{(0)}=-\frac{\lambda}{4 w_{p}^{2}}-\frac{\mathrm{i} e^{2}}{\Theta w_{p}} \cot \left(\frac{\theta_{S}}{2}\right)+\left[\theta_{S} \rightarrow \theta_{S}-\pi\right]
$$

is the exact tree level contribution and the one-loop term, up to order $p^{2} / \mathrm{m}^{2}$, is given by

$$
\begin{aligned}
\mathcal{A}^{(1)} \simeq \frac{m}{8 \pi}( & \left.\frac{\lambda^{2}}{16 m^{4}}-\frac{4 e^{4}}{m^{2} \Theta^{2}}\right)\left[\ln \left(\frac{4 m^{2}}{p^{2}}\right)+\mathrm{i} \pi\right] \\
& -\frac{m}{8 \pi}\left(\frac{3 \lambda^{2}}{32 m^{4}}-\frac{2 e^{4}}{m^{2} \Theta^{2}}\right) \frac{p^{2}}{m^{2}}\left[\ln \left(\frac{4 m^{2}}{p^{2}}\right)+\mathrm{i} \pi\right] \\
& +\frac{m}{8 \pi}\left(\frac{\lambda^{2}}{4 m^{4}}-\frac{14 e^{4}}{3 m^{2} \Theta^{2}}\right)-\frac{m}{8 \pi}\left(\frac{25 \lambda^{2}}{96 m^{4}}+\frac{74 e^{4}}{15 m^{2} \Theta^{2}}\right) \frac{p^{2}}{m^{2}} .
\end{aligned}
$$

One sees that the leading term of the $|\boldsymbol{p}| / m$ expansion of $\mathcal{A}$ coincides with $\mathcal{A}_{\mathrm{NR}}$ if one identifies $v=\lambda / 4 m^{2}$ and chooses $\mu^{2}=4 m^{2}$. Independently of the fixing of $\mu$, by taking $\lambda_{c}^{+}=4 m^{2} v_{c}^{+}$ the one-loop contribution for the leading order (in $|p| / m$ ) vanishes and the tree level one reproduces the $\mathrm{AB}$ scattering. However, the subdominant terms do not vanish for $\lambda=\lambda_{c}^{+}$and constitute additional relativistic corrections to the $\mathrm{AB}$ effect [9], which originate from field theoretical effects such as vacuum polarization and vertex radiative corrections. Notice, in this respect, that the vectorial interaction vertex expressing the bare coupling between the matter and the Chern-Simon fields possesses, in the relativistic case, an energy factor in the zeroth component which is not present in the NR Lagrangian.

\section{Conclusions}

Using a two-component formalism, in this work we have studied perturbatively the first quantized $\mathrm{AB}$ scattering of relativistic scalar particles. We proved that, to eliminate divergences due to the $A^{2}$ coupling, it is necessary to add a contact delta interaction, which, in a field theoretical language, corresponds to a $\left(\phi^{\dagger} \phi\right)^{2}$ self-interaction. Now, it is known that in the fermionic case Pauli's magnetic interaction $\sim \boldsymbol{B} \cdot \psi^{\dagger} s \psi$, with $s$ representing the spin operator, provides the necessary ingredient to make the final result well defined. This immediately suggests that in the scalar case the linearized form $\sigma\left(\phi^{\dagger} \phi\right)$, where in lowest order the external field $\sigma=g \delta(x)$, should be added to the original Lagrangian, as we did. In the NR case the purely magnetic coupling $g \epsilon^{\mu \nu \rho} F_{\nu \rho} j_{\mu}$, where $F_{\nu \rho}$ is the field strength and $j_{\mu}$ the particle's current, equally provides the cancellation of the divergence, but in the relativistic domain it has to be disregarded since it does not have the appropriate momentum dependence and, in the field theory context, it is nonrenormalizable. Comparison between the first-quantized and the field theoretical perturbative expansions shows that the latter has additional contributions from vacuum polarization and vertex radiative corrections. These terms, absent from a direct NR approach, show that the original $\mathrm{AB}$ problem is an idealized situation since vacuum polarization makes the magnetic field necessarily nonvanishing outside the solenoid.

\section{Acknowledgments}

This work was partially supported by CAPES, CNPq and FAPESP, Brazilian agencies.

\section{References}

[1] Aharonov Y and Bohm D 1959 Phys. Rev. 115485 
For a review, see: Ruijsenaars S 1983 Ann. Phys., NY 1461

[2] Berry M V 1980 Eur. J. Phys. 1240

[3] Corinaldesi E and Rafeli F 1978 Am. J. Phys. 461185

[4] Jackiw R 1991 M A B Bég Memorial Volume ed A Ali and P Hoodbhoy (Singapore: World Scientific) Amelino-Camelia G 1994 Phys. Lett. B 326282

Amelino-Camelia G 1995 Phys. Rev. D 512000

Manuel C and Tarrach R 1994 Phys. Lett. B 328113

Ouvry S 1994 Phys. Rev. D 505296

Hagen C R 1995 Phys. Rev. D 522466

Giacconi P, Maltoni F and Soldati R 1996 Phys. Rev. D 53952

[5] Hagen C R 1985 Phys. Rev. D 31848

[6] Jackiw R and Pi S Y 1990 Phys. Rev. D 423500

[7] Bergman O and Lozano G 1994 Ann. Phys., NY 229416

[8] Boz M, Fainberg V and Pak N K 1995 Phys. Lett. A 2071

Boz M, Fainberg V and Pak N K 1996 Ann. Phys., NY 246347

[9] Gomes M, Malbouisson J M C and da Silva A J 1997 Phys. Lett. A 236373

[10] Gomes M, Malbouisson J M C and da Silva A J 1998 Int. J. Mod. Phys. A 133157

[11] Girotti H O and Fonseca Romero F 1997 Europhys. Lett. 37165

[12] Feshbach H and Villars F 1958 Rev. Mod. Phys. 3024

See also: Gross F 1993 Relativistic Quantum Mechanics and Field Theory (New York: Wiley)

[13] Vera F and Schmidt I 1990 Phys. Rev. D 423591

Hagen C R 1990 Phys. Rev. Lett. 64503

Coutinho F A and Peres J F 1994 Phys. Rev. D 492092

Gomes M and da Silva A J 1997 Phys. Rev. D 573579

[14] Gomes M 1998 Topics in Theoretical Physics ed H Aratyn, L A Ferreira and J F Gomes (São Paulo: IFT-UNESP)

[15] Gomes M, Malbouisson J M C and da Silva A J 1996 Mod. Phys. Lett. A 112825 